\title{
Design of Type 2- Interval Fuzzy PID Controller for CSTR
}

\author{
Tejas D. Gangurde \\ Walchand College of Engineering/Department of Electrical Engineering, Sangli, 416415, India \\ Email: tejas.gangurde24@yahoo.co.in \\ Vrushali P. Mahajan \\ Walchand College of Engineering/Department of Electrical Engineering, Sangli, 416415, India \\ Email: vrushalimahajan13@gmail.com
}

Received: 18 September 2018; Accepted: 18 December 2018; Published: 08 October 2019

\begin{abstract}
This paper proposes Type 2- Interval Fuzzy Proportional-Integral-Derivative (T2IFPID) controller for a non-linear system. Type 2- Interval fuzzy logic controller (T2IFLC) is self-possessed in such a way that it is an autonomous process. To decipher the influence, the impression of uncertainty on the controller execution to two different types of curves are outlined i.e. aggressive control curve and smoother control curve. Popov-Lyapunov approach is used to define the stability of the framework.
\end{abstract}

Index Terms - Type 2- Interval Fuzzy, PID, CSTR

\section{INTRODUCTION}

In industries, as a whole, it is realized that the most normally utilized controllers are regular proportionalintegral-derivative controller since they are basic in structure and low in cost [1]. In [2], different proportional-integral-derivative controller tuning strategies are testified i.e. Ziegler and Nichols, Cohen and Coon, Internal Model Control etc. Pole Placement Design Strategies are a portion of the outline systems. The use of proportional, integral and derivative controllers in controlling direct framework may be a successful approach to accomplish wanted execution, yet prop-integ-deriv (i.e. PID) controller would not give a palatable execution when the procedures have a questionable model or if the procedure is non-linear.

In [3] it is determined that Type 1- Interval Fuzzy Logic Controllers(T1IFLC) can be actualized with single or multiple inputs. Despite the fact that the significant research chip away at fuzzy prop-integ-deriv controller fixates is on the customary, either of the two inputs i.e. PI or PD. This sort of controller proposed by Mamdani, in various works. It demonstrates the solitary information that T1IFLC offers additional noteworthy adaptability and better useful properties. In three different sorts, fuzzy controllers are grouped: the gain scheduling, direct action (DA) sort, and a mix of DA and gain scheduling sorts. The DA sort generally utilized as a part of fuzzy propinteg-deriv controller application; here in the criticism control circle fuzzy prop-integ-deriv controller is added, and the prop-integ-deriv controller activities computed utilizing fuzzy derivation. In gain scheduling sort controllers, singular prop-integ-deriv controller pick-ups are figured through fuzzy deduction.

As of late, the fundamental research concentrates on T2IFLC [4]. For the most part, T2IFLC finish prevalent exhibitions as it gives an extra level of the opportunity given by the impression of vulnerability in their Type-2 Interval Fuzzy Sets (T2IFS). Key contrasts between T2IFLC and T1IFLC are adaptive-ness, implying that the installed Type 1- Interval Fuzzy Sets (T1IFSs) used to register the limits of the type deduced interval change as input varies. The upper-side membership functions (UMF) and lower-side membership functions (LMF) of a similar T2IFS might be utilized at the same time in processing each bound of the type deduced interval. T1IFLC lack these properties, because of this a T1IFLC is unable to execute the intricate control surface of a T2IFLC utilizing a similar rule base. Interior structure of the T2IFLC is similar to T1IFLC. Principle distinction is desired fuzzy membership function (FM), yet advanced algorithms are developed for designing T2IFLC for generating the control surface [5]. The primary weakness of this approach is that it does not clarify how the footprint of uncertainty parameters influences the execution and robustness of the T2IFLC [6]. In this way, determining the scientific structure of a T2IFLC in the system of the nonlinear control may be an effective approach to look at it $[7,8]$. However, the orderly outline and strength examination of the T2IFLC are yet difficult issues because of its generally more intricate structure $[9,10]$. Conventional PID is not able to give general solution to all control problems because the processes involved are in general complex and with delays, nonlinear, timevariant, non-stationary, and often with poorly defined dynamic. To overcome these difficulties, various types of modified fuzzy controllers are developed. In [12,13] T2IFPID controller and Popov Lyapunov method for stability analysis is presented which gives better performance as compare to traditional prop-integ-deriv controller. 


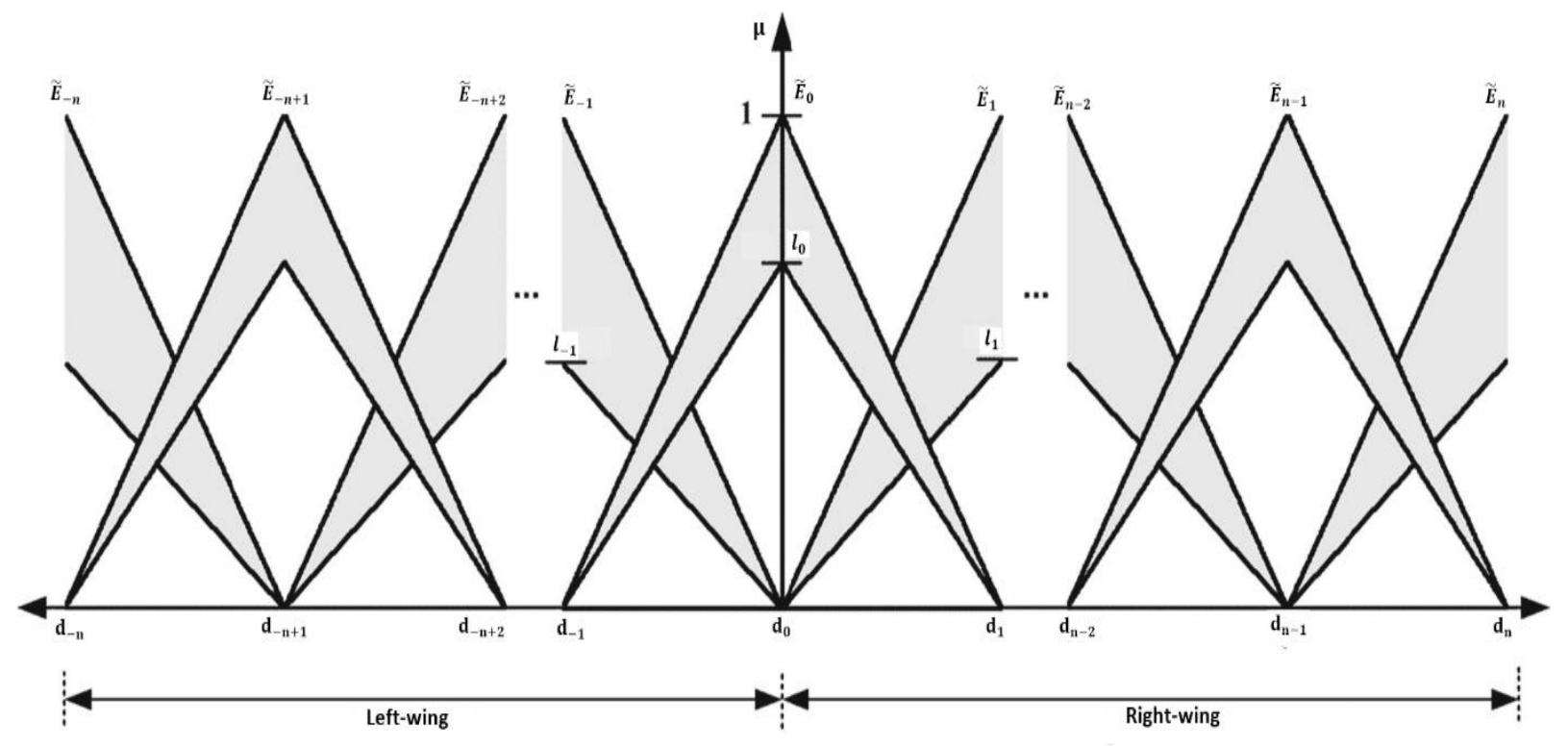

Fig.1. Demonstration of Type-2 Interval triangular membership function for error

\section{T2IFLC STRUCTURE}

In this segment, the proposed T2IFLC structures inputoutput mapping is determined. For effortlessness, the antecedent part for the T2IFLC FM of the fuzzy rule base is presented by consistently conveyed symmetrical triangular. From the error $(e)$ i.e. the linguistic value of the input are indicated as $\overline{\tilde{E}}_{i}$ where $\mathrm{i}=\{-\mathrm{n},(-\mathrm{n}+1),(-$ $\mathrm{n}+2), \ldots,-2,-1,0,1,2, \ldots,(\mathrm{n}-2),(\mathrm{n}-1), \mathrm{n}\}$. The presented type-2 fuzzy set $\left(\tilde{E}_{i}\right)$ is referred as LMF $\left(\mu \tilde{E}_{i}\right)$ and $\operatorname{UMF}\left(\bar{\mu} \tilde{E}_{i}\right)$. As appeared in Fig.1, the height of the LMF is represented by $l_{n}$ though the center of the $\tilde{E}_{i}$ is indicated by $d_{n}$. The input T2IFSs are symmetrical, subsequently, the information space $(\underline{e})$ is separated in two fundamental districts which are named as the Left wing $\left(\underline{e} \in\left[\begin{array}{ll}d_{-n} & d_{0}\end{array}\right]\right)$ and Right wing $\left(\underline{e} \in\left[\begin{array}{ll}d_{0} & d_{n}\end{array}\right]\right)$. In addition, following properties are controlled by an additional type reduction system since T2IFLC utilize and process T2IFSs. A few investigations have been introduced to examine the impact of the footprint of uncertainty and additional type reduction process on wanted fuzzy enrolment (i.e., control surface) [5]. The type-2 interval fuzzy mapping [4], characterized T2IFLC membership functions:

(i) $\bar{\mu} \tilde{E}_{i}(\underline{e})+\bar{\mu} \tilde{E}_{i+1}(\underline{e})=1, \mathrm{i}=-\mathrm{n}, . .,-2,-1,0,1,2, \ldots, \mathrm{n}$

(ii) $\mu \tilde{E}_{i}(\underline{e})=l_{i}^{*} \bar{\mu} \tilde{E}_{i}(\underline{e}),-\mathrm{n}, .,-2,-1,0,1,2, \ldots, \mathrm{n}$

(iii) $\bar{l}_{-i}=l_{i}, \mathrm{i}=1,2,3, \ldots, \mathrm{n}$

The proposed T2IFLC run development is described as:

$$
r_{i}: \operatorname{IF}(\underline{e}) \text { is } \tilde{E}_{i} \operatorname{THEN}\left(\underline{e}_{f u z}^{\prime}\right) \text { is } B_{i}, \mathrm{i}=1,2, \ldots \ldots, \mathrm{N}
$$

The aggregate total sum of rule numbers is given as $\mathrm{N}$ $=2 \mathrm{n}+1$, while the resultant part deciphers the crisp singleton esteems $\left(B_{i}\right)$ which are consistently disseminated in the scope of $[-1,1]$. Liang and Mendel revealed that the defuzzified output of a T2IFLC can be computed as:

$$
\underline{e}_{f u z}=\frac{\underline{e}_{f u z}^{l \prime}+\underline{e}_{f u z}^{r \prime}}{2}
$$

where $\left(\underline{e}^{r \prime} f u z\right)$ and $\left(\underline{e}^{l \prime} f u z\right)$ projects the endpoints of the type deduced set.

Moreover $\left(\underline{e}_{f u z}^{r \prime}\right),\left(\underline{e}_{f u z}^{l^{\prime}}\right)$ are calculated as below:

$$
\begin{gathered}
\underline{e}_{f u z}^{r \prime}=\frac{\sum_{j=1}^{R} \mu \tilde{E}_{i}(\underline{e}) * B_{i}+\sum_{j=R+1}^{N} \bar{\mu} \tilde{E}_{i}(\underline{e}) * B_{i}}{\sum_{j=1}^{R} \underline{\mu}_{i}(\underline{e})+\sum_{j=R+1}^{N} \bar{\mu} \tilde{E}_{i}(\underline{e})} \\
\underline{e}_{f u z}^{l \prime}=\frac{\sum_{j=1}^{R} \bar{\mu} \tilde{E}_{i}(\underline{e}) * B_{i}+\sum_{j=R+1}^{N} \underline{\mu}_{i}(\underline{e}) * B_{i}}{\sum_{j=1}^{R} \bar{\mu} \tilde{E}_{i}(\underline{e})+\sum_{j=R+1}^{N} \underline{\mu}_{i}(\underline{e})}
\end{gathered}
$$

Here, (R, L) is the emerging set that decreases/ increases separately. T2IFLC completely covers the triangular T2IFSs in the feeling of upper and lower FM's. Subsequently, it can be constantly ensured that a crisp estimation of $\underline{e}$ has a place with two progressive T2IFSs, i.e. $\widetilde{E}_{i}$ and $\widetilde{E}_{i+1}$. Subsequently, for any crisp information, simply two rules $(\mathrm{N}=2)$ are constantly operated as the points $(\mathrm{R}, \mathrm{L})$ are constantly equivalent to "1". A triangular shape FM of T2IFLC is obtained in the form of LMF and UMF. The reduced type set are determined as: 


$$
\begin{aligned}
\underline{e}_{f u z}^{r \prime} & =\frac{\mu \tilde{E}_{i}(\underline{e}) * B_{i}+\bar{\mu} \tilde{E}_{i+1}(\underline{e})^{*} B_{i+1}}{\tilde{E}_{i}(\underline{e})+\bar{\mu} \tilde{E}_{i+1}(\underline{e})}, \\
\underline{e}_{f u z}^{l \prime} & =\frac{\bar{\mu} \tilde{E}_{i}(\underline{e})^{*} B_{i}+\underline{\mu} \tilde{E}_{i+1}(\underline{e}) * B_{i+1}}{\bar{\mu} \tilde{E}_{i}(\underline{e})+\underline{\mu} \tilde{E}_{i+1}(\underline{e})}
\end{aligned}
$$

After replacing (5) in (2), proposed T2IFLC closed form mapping is obtained. Following are the properties of the presented a T2IFLC output.

(i) In relation to ' $\underline{e}$ ', ' $\underline{e}_{f u z}^{\prime}$ ' has a continual function.

(ii) In relation to the input ' $\underline{e}$ ', ' $\underline{e}_{f u z}^{\prime}$ ' is proportional, i.e. $\underline{e}_{f u z}^{\prime}(e)=-\underline{e}_{f u z}^{\prime}(\underline{-e})$.

(iii) In an event that if an input error equivalent to zero then fuzzified error equivalents to be zero. It is mandatory to have zero error i.e. $(\underline{e})=0$ then $\underline{e}_{f u z}^{\prime}=0$

\section{DESIGN STRATEGy For T2IFPID CONTROLlER}

In this section, the outlined procedure for singular input T2IFLC is presented. The output is expressed in the error $(\underline{e})$ domain since the T2IFLC has singular input. This clarifies the design method of T2IFLC to the generation of non-linear control curve. In this strategy, the height $\left(l_{i}\right)$ of lower-side FM's of $\widetilde{E}_{i}$ are considered as design parameters. Firstly, design parameters effect on T2IFLC are scrutinised and then an autonomous design method is proposed to generated control curves/curves. The parameters for T2IFLC structure are set as $\underline{B}_{-1}=$ $-1, \underline{B}_{+1}=+1, \underline{B}_{0}=0, d_{-1}=-1, d_{+1}=+1$ and $d_{0}=0$ [11]. For the information Left-wing $(\underline{e} \in[-1,0])$, the end purposes of the type decreased set would then be able to be inferred as takes after:

$$
\begin{aligned}
\underline{e}_{f u z}^{l \prime} & =\frac{-\bar{\mu} \tilde{E}_{-1}(\mathrm{e})}{\bar{\mu} \tilde{E}_{-1}(\mathrm{e})+\mu \tilde{E}_{0}(\mathrm{e})}, \\
\underline{e}_{f u z}^{r \prime} & =\frac{-\bar{\mu} \tilde{E}_{-1}(\mathrm{e})}{\underline{\mu} \tilde{E}_{-1}(\mathrm{e})+\bar{\mu} \tilde{E}_{0}(\mathrm{e})}
\end{aligned}
$$

For the Right-wing $(\underline{e} \in[0,+1])$, the end point of the type deduced sets reduces to:

$$
\begin{gathered}
\underline{e}^{l \prime}{ }_{f u z}=\frac{\underline{\mu} \tilde{E}_{1}(\underline{e})}{\bar{\mu} \tilde{E}_{0}(\underline{e})+\mu \tilde{E}_{1}(\underline{e})}, \\
\underline{e}_{f u z}^{r \prime}=\frac{\underline{\mu \tilde{E}_{1}(\underline{e})}}{\underline{\mu} \tilde{E}_{0}(\underline{e})+\bar{\mu} \tilde{E}_{1}(\underline{e})}
\end{gathered}
$$

Table 1. Expressions of $\underline{e}_{f u z}^{l}$ and $\underline{e}_{f u z}^{r}$

\begin{tabular}{cll}
\hline & Left wing & Right wing \\
& $' \underline{e}$ ' $\in[-1,0]$ & 'e $\underline{\underline{e} \in[0,+1]}$ \\
\hline$\underline{e}^{l \prime} f u z$ & $\frac{-\bar{\mu} \tilde{E}_{-1}(\underline{e})}{\bar{\mu} \tilde{E}_{-1}(\underline{e})+\underline{\mu} \tilde{E}_{0}(\underline{e})}$ & $\frac{\underline{\mu} \tilde{E}_{1}(\underline{e})}{\bar{\mu} \tilde{E}_{0}(\underline{e})+\underline{\mu} \tilde{E}_{1}(\underline{e})}$ \\
\hline$\underline{e}^{r \prime} f u z$ & $\frac{-\bar{\mu} \tilde{E}_{-1}(\underline{e})}{\mu \tilde{E}_{-1}(\underline{e})+\bar{\mu} \tilde{E}_{0}(\underline{e})}$ & $\frac{\underline{\mu} \tilde{E}_{1}(\underline{e})}{\mu \tilde{E}_{0}(\underline{e})+\bar{\mu} \tilde{E}_{1}(\underline{e})}$ \\
\hline
\end{tabular}

To inspect the impact of the design parameters $\left(l_{-1}, l_{0}, l_{1}\right)$ on the output effectively the inferred explanatory articulations of $\underline{e}_{f u z}^{l \prime}$ and $\underline{e}_{f u z}^{r \prime}$ for a "three rule" interval T2IFLC are organized in Table 1. In this examination, the just Right-wing will be assessed in detail. Because of symmetrical and uniformly distributed nature of input and output FM, the evaluation of Rightwing can be extended for Left-wing in detail. The accompanying meta-rules inferred to shape a control activity from the determined articulations of $\underline{e}_{f u z}^{l \prime}$ and $\underline{e}_{f u z}^{r \prime}$ for Right-wing, to get a palatable framework execution.

a. The values of ' $\underline{e}^{l \prime} f u z$ ' decrements/increments, if the values of $\bar{\mu} \tilde{E}_{1}(\underline{e})$ (i.e. $\left.l_{1}\right)$ decrements/increments respectively.

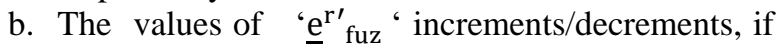
the values of $\bar{\mu} \widetilde{\mathrm{E}}_{0}(\mathrm{e})$ (i.e. $\mathrm{l}_{0}$ ) decrements/increments respectively. The defuzzified output of a T2IFLC $\left(\underline{\mathrm{e}}^{\prime}\right.$ fuz $)$ is the mean value of ' $\underline{\mathrm{e}}^{\mathrm{r}}$ 'fuz' and ' $\underline{\mathrm{e}}^{\mathrm{l}}$ fuz ' values.

c. If the values of ' $l_{1}$ ' is incremented while ' $l_{0}$ ' is decremented then the values of ' $\underline{e}_{f u z}^{\prime}$ ' is incremented since the values of both ' $\underline{e}^{r \prime}{ }_{f u z}$ ' and ' $\underline{e}^{l \prime} f u z$ ' are incremented. Hence, we obtain an aggressive control action.

d. If the values of ' $l_{1}$ ' is decremented while ' $l_{0}$ ' is incremented then the values of ' $\underline{e}_{f u z}^{\prime}$ ' is decremented since the values of both ' $\underline{e}^{r \prime}{ }_{f u z}$ ' and ' $\underline{e}^{l \prime} f u z$ ' are decremented. Hence, we obtain a smooth control action.

By choosing $l_{1}$ moderately greater then $l_{0}$, a forceful nonlinear control activity can be produced i.e. 1 $\geq l_{1}=l_{-1} \geq l_{0} \geq 0$. To get aggressive control curve, selecting $\underline{m}_{1}=\underline{m}_{-1}=0.9$ and $\underline{m}_{0}=0.2$, the control curve showed in Fig.2. At the point when $\underline{\underline{e}}$ is near zero, the control curve has a high affect ability. For quick transient framework response, aggressive control curve is favoured. At set point value, i.e. $\underline{e}$ equals to zero aggressive control action is sensitive to noises.

Smooth control action is acquired by choosing $l_{0}$ moderately greater than $l_{1}$, i.e. $1 \geq l_{0} \geq l_{1}=l_{-1} \geq 0$. To acquire smooth control curve given in Fig. 3, selecting $l_{1}=l_{-1}=0.2$ and $l_{0}=0.9$. At the point when $e$ is near to zero, it has low affectability. For robust control performance against parameter vulnerabilities and additionally background noises, smooth control curve is favoured.

The secondary part of proposed controller structure is a traditional prop-integ-deriv controller. The control law for T2IFPID controller is given by

$$
\mathrm{u}(\mathrm{t})=K_{P}(t) \underline{e}_{f u z}(t)+K_{I}(t) \int \underline{e}_{f u z}(t)+K_{D} \frac{d \underline{e}_{f u z}(t)}{d t}
$$

where,

$K_{P}=$ Proportional gain

$K_{D}=$ Derivative gain

$K_{I}=$ Integral gain 


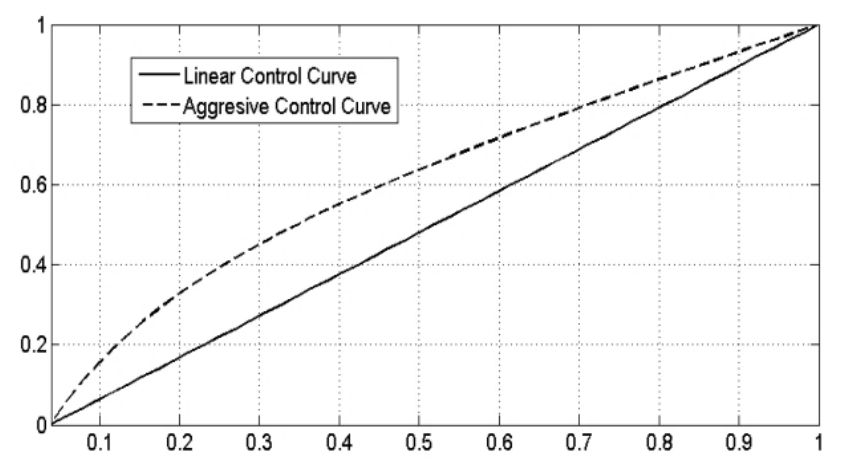

Fig.2. Linear control curve versus Aggressive control curve

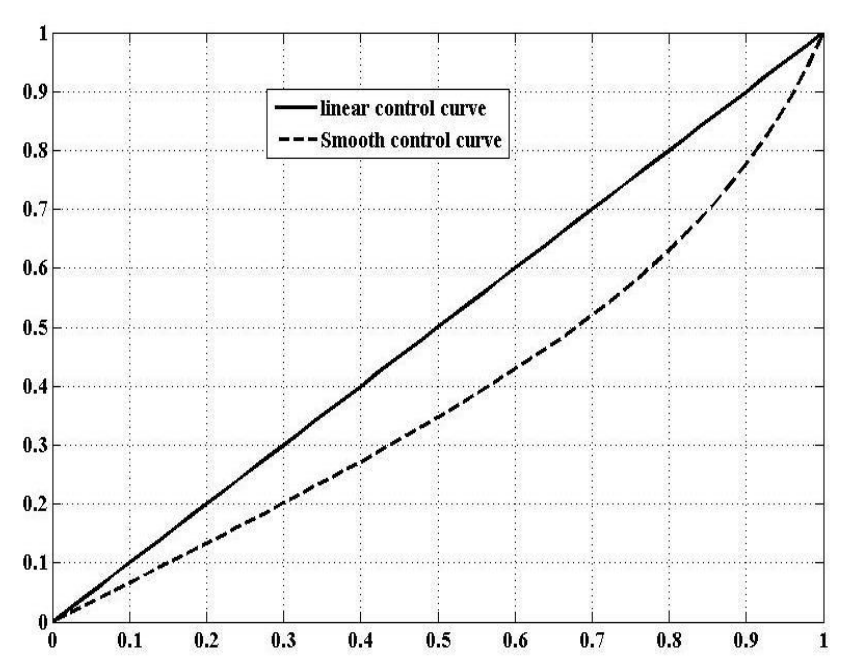

Fig.3. Linear control curve versus Smooth control curve

\section{StABILITy ANALYSIS}

Robust Stability Analysis for fuzzy based systems is carried out through Popov-Lyapunov method [13]. In this approach initially, expand a nonlinear system into a Taylor series. As presented in [13] the system is described in terms of Perturbed Lur'e system. Following are the steps to determine the robust stability.

1. Plot the Popov's plot for the nominal system. If the Popov's criteria are pleased, then find the significant slope $(r)$. The $r$ should be scalar such that value of $r>0$.

2. Calculate ' $v$ ' and ' $\gamma$ ' from [13].

3. Choose a symmetric positive-definite matrix $E$ and a positive real number ' $\epsilon$ ', then the ' $P$ ' matrix is acquired by solving the following Ricatti equation:

$$
\underline{A}_{r}^{T} \underline{P}+\underline{P A}_{r}-\underline{P R}_{r}+\underline{Q}_{r}=0
$$

where

$$
\underline{A}_{r}=A-\frac{1}{\gamma} b v^{T}, \underline{Q}_{r}=\epsilon E+\frac{v v^{T}}{\gamma}, \underline{R}_{r}=-\frac{b b^{T}}{\gamma}
$$

4. Choose a positive-definite matrix ' $E_{0}$ ' such that $\epsilon E=\epsilon E_{0}+\boldsymbol{\delta}$ I, then the proper value of ' $\boldsymbol{\delta}$ ' can be obtained.

5. ' $\beta$ ' i.e. measurement of robustness can be obtained can be obtained from inequality [13].

\section{Simulation Result AND Stability ANALysis}

For calculating transfer function of CSTR cooling process, the step response is taken into consideration. For the step response, the input is step input, the initial temperature is taken as $57^{\circ} \mathrm{C}$ and the set point is taken as $45^{\circ} \mathrm{C}$. The process has very large dead time and is highly damped. Therefore, the step response can be fitted into a simple first-order model with dead-time.

Therefore, the transfer function of the CSTR process is given by

$$
\underline{G}(s)=\frac{0.12 e^{-2 s}}{3 s+1}
$$

Tuning of prop-integ-deriv for CSTR is done by Ciancone correlation [12], it is resulting as follows: $K_{P}=6.667, K_{D}=2$ and $K_{I}=1.9$. Simulation result of CSTR is shown in fig.4 has comparison between T2IFPID with aggressive, smooth control action and PID controller. Initially unit step input is applied to the process. It can be seen in Fig. 4 and Fig. 5, three different types of controller are able to track the reference with different rise time and settling time. Response due to Aggressive control action Fuzzy controller has less rise time compared to other controller; while response due to Smoother control action Fuzzy controller has higher settling time compared to other controller performance. Table 2 shows step response characteristics of prop-integderiv controller, T2IFPID controller (Smooth) and T2IFID controller (Aggressive); where unit step input is applied to the process.

To find out the stability of fuzzy system using PopovLyapunov method first system is converted into perturbed Lur'e system [13], which is represented by transfer function as follows-

$$
\begin{aligned}
& G_{S-I T 2}(s)=\frac{-0.1067 S+0.32}{S^{2}+1.269 S+0.525} \\
& \underline{G}_{A-I T 2}(s)=\frac{-0.1067 S+0.32}{S^{2}+1.226 S+0.653}
\end{aligned}
$$




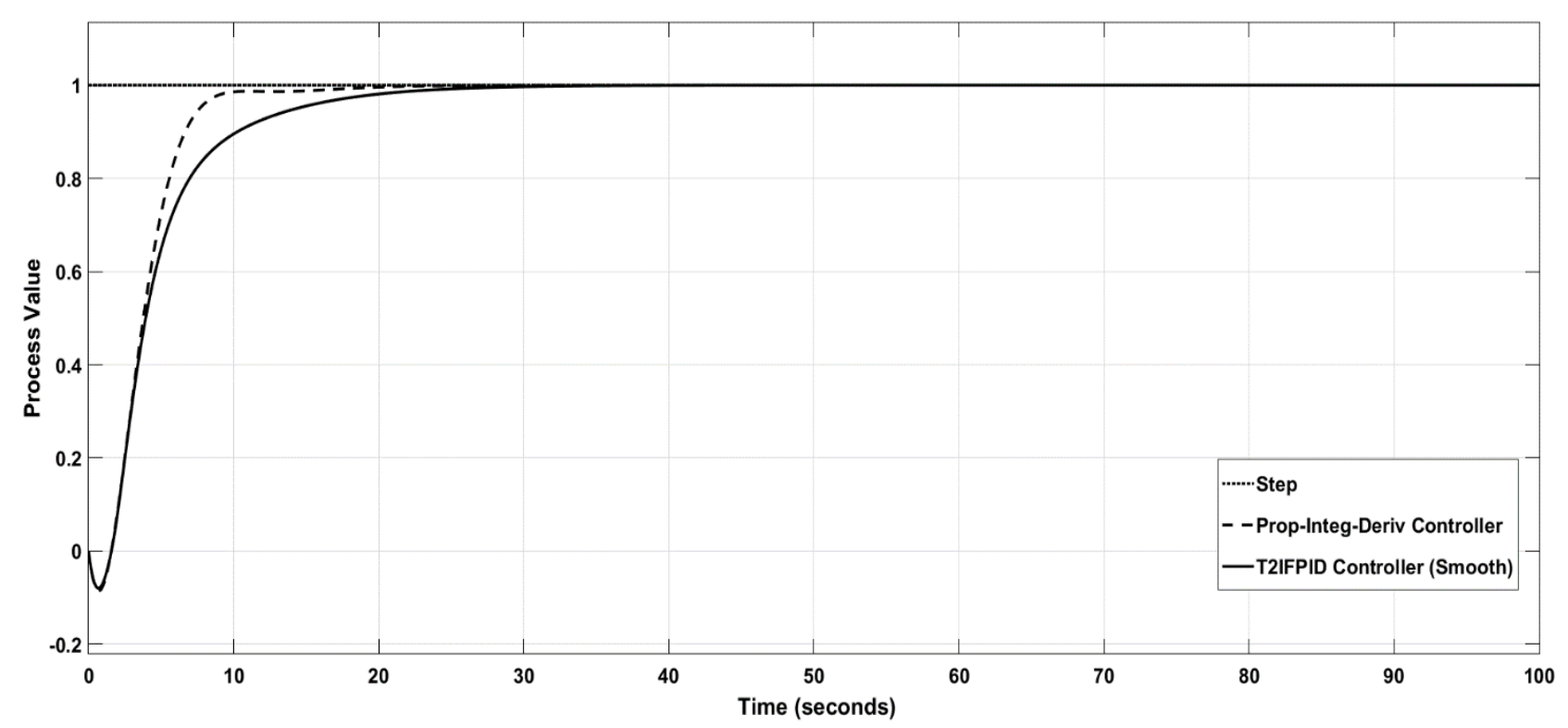

Fig.4. Setpoint tracking of T2IFPID (smooth) controller and Prop-Integ-Deriv controller

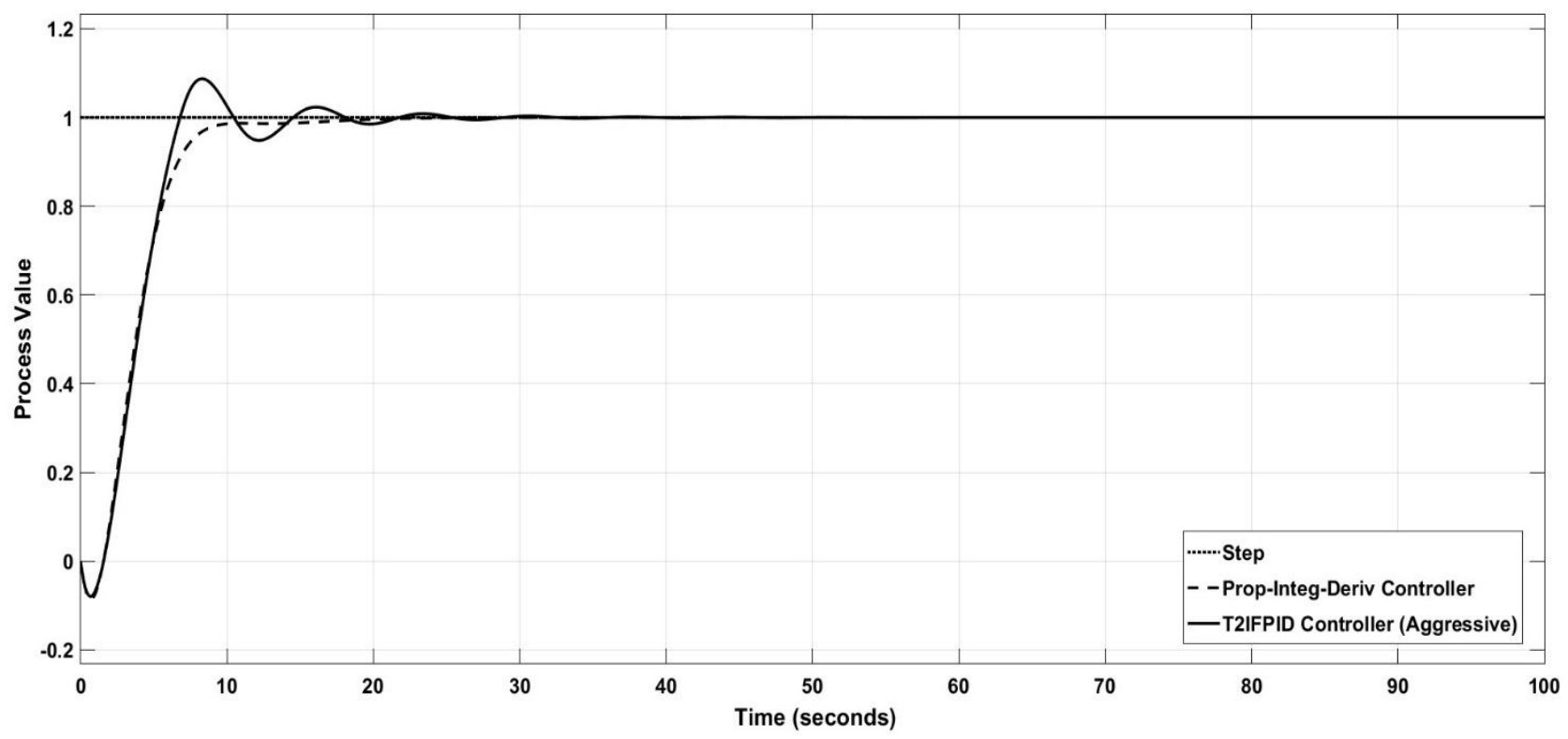

Fig.5. Setpoint tracking of T2IFPID (aggressive) controller and Prop-Integ-Deriv controller

Table.2. Step response characteristic of continuous stirred tank reactor with the controller

\begin{tabular}{llll}
\hline & $\begin{array}{l}\text { Prop-Integ- } \\
\text { Deriv } \\
\text { controller }\end{array}$ & $\begin{array}{l}\text { T2IFPID } \\
\text { controller } \\
\text { (Smooth) }\end{array}$ & $\begin{array}{l}\text { T2IPID } \\
\text { controller } \\
\text { (Aggressive) }\end{array}$ \\
\hline $\begin{array}{l}\text { Rise } \\
\text { Time(sec.) }\end{array}$ & 4.540 & 7.913 & 4.195 \\
\hline $\begin{array}{l}\text { Settling } \\
\text { Time(sec.) }\end{array}$ & 42.273 & 32.668 & 36.972 \\
\hline $\begin{array}{l}\text { Overshoot } \\
(\%)\end{array}$ & 0.000 & 0.502 & 8.152 \\
\hline IAE & 4.386 & 5.488 & 4.585 \\
\hline
\end{tabular}

Transfer function $\underline{G}_{S-I T 2}(s)$ represent perturbed Lur'e system of fuzzy system which implement smoother control action while Transfer function $\underline{G}_{A-I T 2}(s)$ represent perturbed Lur'e system of fuzzy system which implement aggressive control action. Fig. 6 represent popov plot of the system represented by transfer function (14), from that plot it can be concluded as given system satisfies popov criterion for slope of line $r \geq 1.45$. Similarly, popov plot, of system represented by (15) is shown in Fig. 7, from that it can be seen that given system satisfies popov criterion for slope of line $r \geq 1.44$. Appling Lyapunov stability method $\underline{P}$ matrices are given as follows-;

$$
\begin{aligned}
\underline{P}_{S-I T 2} & =\left[\begin{array}{cc}
1.4766 & -0.1342 \\
-0.1342 & 0.4102
\end{array}\right] \\
\underline{P}_{A-I T 2} & =\left[\begin{array}{cc}
1.3204 & -0.3408 \\
-0.3486 & 0.2845
\end{array}\right]
\end{aligned}
$$

Given $\underline{P}$ matrices are symmetric positive-definite, from this popov criterion and Lyapunov stability method its can be concluded that equilibrium point zero is uniformly 
asymptotically stable. Robustness measure $\underline{\beta}$ can be calculated using [13], which are $\underline{\beta}_{S-I T 2}=0.7015$ for smoother control action fuzzy system and $\underline{\beta}_{A-I T 2}=$ 0.4627 for aggressive control action fuzzy system. From this it is stated that smoother control action fuzzy system is more robust than aggressive control action fuzzy system.

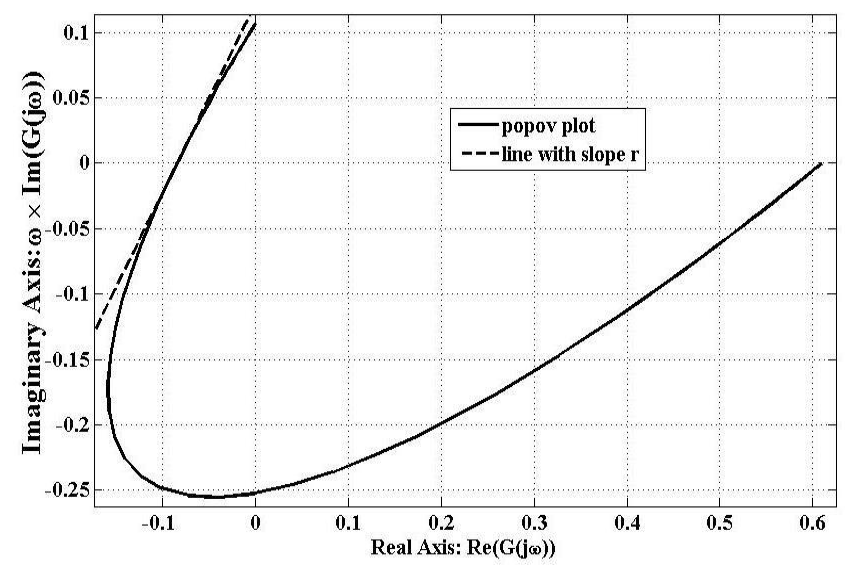

Fig.6 Popov plot of $\mathrm{G}_{\mathrm{S}-\mathrm{IT} 2}(\mathrm{~s})$

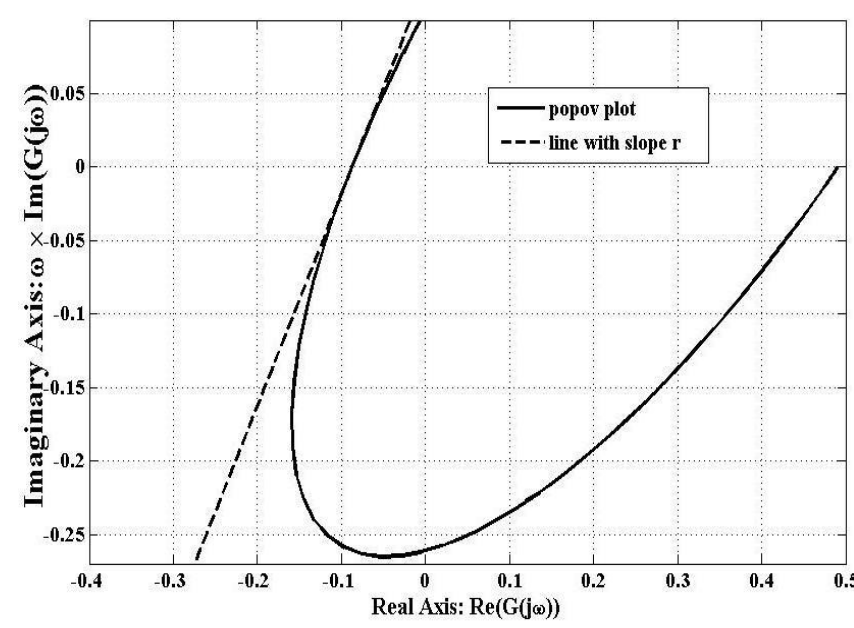

Fig.7 Popov plot of $\mathrm{G}_{\mathrm{A}-\mathrm{IT} 2}(\mathrm{~s})$

\section{CONCLUSION}

The proposed design method is the simplest method to design T2IFLC. The proposed controller possesses the properties of Prop-Integ-Deriv controller. The T2IFLC controller output is completely expressed in error. Hence, an autonomous design method is proposed to generate nonlinear control curves. The performance of the T2IFLC is compared with different controllers to investigate transient state performance and disturbance rejection.
Stability analysis is carried through Popov-Lyapunov method to define the robustness of the proposed controller.

Aggressive control action is favored for quick transient reaction since it is delicate to the commotion, particularly around setpoint esteem. At relentless state, controller, which has a smooth control surface, is conceivably more powerful against non-linearity and vulnerabilities. T2IFLC gives a diverse reaction to the various footprint of uncertainty parameters. T2IFPID controller beats the prop-integ-deriv controller.

\section{REFERENCES}

[1] KJ Åström, T Hägglund, The future of PID control, Control Engg. Practice 9 (11) (2001) 1163-1175.

[2] KJ Åström, T Hägglund, PID controllers: Theory, Design and Tuning, Instrument Soc. of America, Research Triangle Park, NC, 1995.

[3] G. Mann, Bao-Gang Hu, R. Gosine, "Analysis of direct action fuzzy PID controller structures," IEEE Trans. on Syst., vol. 29, no. 3, pp. 371-388, Jun.1999.

[4] D. Wu, "On the fundamental differences between interval type-2 and type-1 fuzzy logic controllers," IEEE Trans. on Fuzzy Syst., vol. 20, no. 5, pp. 832-848, Oct. 2012.

[5] O. Castillo, R. Martinez-Marroquin, P. Melin, F. Valdez, J. Soria, "Comparative study of bio-inspired algorithms applied to the optimization of type-1 and type-2 fuzzy controllers for an autonomous mobile robot," Info Sciences, vol. 192, pp. 19-38, June 2012.

[6] X. Du and H. Ying, "Derivation and analysis of the analytical structures of the interval type-2 fuzzy-PI and PD controllers," IEEE Trans. on Fuzzy Syst., vol. 18, no. 4, pp. 802-814, Aug. 2010.

[7] M. Nie and W. Tan, "Analytical structure and characteristics of symmetric Karnik-Mendel type-reduced interval type-2 fuzzy PI and PD controllers." IEEE Trans. on Fuzzy Syst., vol. 20, no. 3, pp. 416-430, June 2012.

[8] H. Zhou and H. Ying, "A method for deriving the analytical structure of a broad class of typical interval type-2 mamdani fuzzy systems," IEEE Trans. on Fuzzy Syst., vol. 21, no. 3, pp. 447-458, June 2013.

[9] M. Biglarbegian, W. Melek and J. Mendel, "On the stability of interval type-2 TSK fuzzy logic control systems," IEEE Trans. on Fuzzy Syst., vol. 40, no. 3, pp. 798-818, June 2010.

[10] H. Lam and L. Seneviratne, "Stability analysis of interval type-2 fuzzy-model-based-control systems," IEEE Trans. on Syst., Man, and Cybernetics, Part B (Cybernetics), vol. 38, no. 3, pp. 617-628, June 2008.

[11] T. Kumbasar, "A simple design method of interval type-2 fuzzy PID controllers," Soft Comput., vol. 18, no. 7, pp. 1293-1304, 2014

[12] T. Marlin, "Process control designing processes and control systems for dynamic performance," ch. 9, pp. 279281.

[13] T. Kumbasar, "Robust stability analysis of PD type single input interval type-2 fuzzy control systems," Int. Conf. on Fuzzy Systems, July 2014. 


\section{Authors' Profiles}

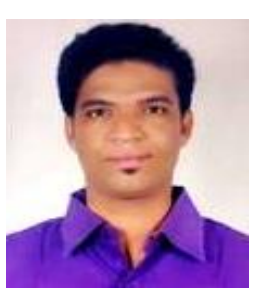

Tejas D. Gangurde completed Master of Technology in Electrical Control Systems from Walchand College of Engineering, Sangli, India-416415. Completed Bachelor of Electrical Engineering from University of Pune in 2014.

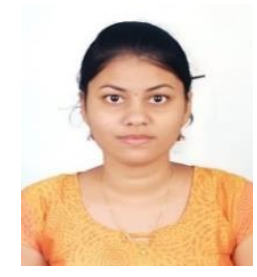

Vrushali P. Mahajan currently pursuing Master of Technology in Electrical Control Systems from Walchand College of Engineering, Sangli, India-416415.

How to cite this paper: Tejas D. Gangurde, Vrushali P. Mahajan, "Design of Type 2- Interval Fuzzy PID Controller for CSTR", International Journal of Image, Graphics and Signal Processing(IJIGSP), Vol.11, No.10, pp. 23-29, 2019.DOI: $10.5815 /$ ijigsp.2019.10.04 